\title{
Identification of people with disabilities using participatory rural appraisal and key informants: A pragmatic approach with action potential promoting validity and low cost
}

\author{
JOSEPH K. GONA ${ }^{1}$, TENGBIN XIONG ${ }^{2}$, MOHAMMAD A. MUHIT ${ }^{3}$, CHARLES R. NEWTON $^{4}$, \\ and SALLY HARTLEY ${ }^{2}$ \\ ${ }^{1}$ Kenya Medical Research Institute, Centre for Geographic Medicine Research (Coast), Kilifi, \\ Kenya \\ 2 University of East Anglia, School of Allied Health Professions, Norwich, UK \\ ${ }^{3}$ London School of Hygiene and Tropical Medicine, Department of Infectious and Tropical \\ Diseases, International Centre for Eye Health, Clinical Research Unit, London, UK \\ ${ }^{4}$ University College London, Institute of Child Health, Neuroscience Unit, London, UK
}

\begin{abstract}
Background-Surveys have been the conventional methods used for identification of people with disabilities; however, they have been observed to be expensive and time-consuming that may not be affordable or practical. As a result, the participatory rural appraisal (PRA) and key informant (KI) approaches have been developed and increasingly used in the resource-poor countries.
\end{abstract}

Objective-To investigate the strengths and weaknesses of PRA and KI approaches in the identification of people with disability in resource-poor countries.

\begin{abstract}
Method-A review of published related papers was performed by searching electronic databases including PubMed, Scirus, Health on the Net (HON), Ovid Medline and SOURCE disability database.
\end{abstract}

Results-A total of 11 relevant papers were identified from the literature that used PRA or KI methods or both. The PRA and KI approaches were not only consistently less expensive than conventional surveys, but also observed to be simple and fast for identifying disabilities according to local perceptions, although they were less sensitive. The evidence showed that PRA and KI processes had the benefit of engaging and developing long-term partnerships with the local communities and so the likelihood of positive long-term impact on the community.

Conclusions-The PRA and KI approaches could be fast and cost-effective methods for identifying people with disabilities as an alternative to surveys. They are especially useful when identification is related to subsequent development of community-based services for persons with disabilities. However, surveys were shown to be more sensitive and therefore more accurate for establishing prevalence rates of impairment.

\section{Keywords}

Participatory rural appraisal; key informant; identification of people with disabilities 


\section{Introduction}

Disability has been defined as a dynamic multi-dimensional interaction between the key domains of impaired body structure and function, activity limitations, participation restrictions and contextual factors [1]. The International Classification of Functioning, Disability and Health (ICF) puts more emphasis on function than on the condition or disease in terms of disability itself [1].

Surveys have been the conventional methods used for the identification of people with disabilities in several studies conducted in resource-poor countries [2-5]. They have been observed to be expensive and time-consuming, though their sensitivity in the identification of disability has been established [6-8]. For example in a comparative study of key informant and survey methods conducted in Bengal, the cost of identifying one case was US $\$ 11$ for the key informant approach and US\$14 for the survey [9]. Also in Kilifi, Kenya, the cost of finding one case was estimated to be US $\$ 1.20$ for the participatory rural appraisal (PRA) and US\$7 for a house-to-house survey conducted in the same area $[5,10]$.

PRA is a collection of evaluative methods that enables community members to share and enhance their experiences and then set priorities on how to change these situations [11,12]. It is a defined methodology and systematic learning process that engages and empowers the local people to take control of their own situations [13]. It was first used in the agriculture and public health sectors to promote development within rural communities. More recently, this approach has been used in other sectors such as health [14] and disability [10,15,16]. In PRA, emphasis is on the community's perceptions of disability and their participation in the process of identification. Social mapping, seasonal calendar, historical profiles and timelines, wealth ranking and Venn diagrams are among the tools used in this approach.

Key informant (KI) approaches utilise community members who are knowledgeable about the topic of interest and willing to share this knowledge. In this context, these are people who live and work in their local community, who have a social role through their vocation and who therefore, know the local context as well as the people about whom information is being sought. KI surveys have been described as an alternative to population-based studies in evaluating community-based programmes [17], needs assessments [18], studies of mental health [19], epilepsy [9,20] and AIDS programmes [21]. The key informants described in these surveys are of three categories: 'economic dominants' that is individuals who occupy major economic roles in the community; 'prescribed influentials' that is community leaders who hold positions formally designed to sanction and facilitate influence in the community, such as civic leaders; and 'attributed influentials' that is community members who are perceived by others as being influential in community decision making [17].

The PRA and KI approaches are similar in that they utilise local people from the community and could use anybody regardless of educational level or socioeconomic status. However, PRA is mostly activity-oriented, whereas KI is based on interviews. The participants in this review article were predominantly village leaders, village women groups, district social welfare department field staff, community level non-governmental development organisation (NGDO) staff, health workers from both government and NGDO sectors, schools teachers, imams, journalists and students.

This article will focus on the application of PRA and KI approaches for identification of people with disabilities in the resource-poor countries. 


\section{Methods}

We included primary studies that used PRA or KI as research methods and provided the data on validity or cost. Referring to the guidance from the NHS Centre for Reviews and Dissemination [22], we identified publications by searching electronic databases, scanning reference list of papers and consulting experts in the specialty. We found no other reviews focusing on the applications of PRA and KI methods in the resource-poor countries. The electronic databases we have searched included PubMed, Ovid Medline, Health on the Net Foundation (HON), Scirus and SOURCE disability database. The main search terms used were 'participatory rural appraisal', 'key informants', 'disabilities', 'identification', 'identif $\$$ ', and 'prevalence'. No language limits were placed on the searches.

One reviewer (JG) examined titles, abstracts and key words of citations, as given on electronic databases, and selected those which mentioned the concept of 'participatory rural appraisal' or 'key informant'. Where possible the full text of all of potentially relevant citations was then obtained. All retrieved references were exported to a reference management database by using EndNote $v 9.0$ (Thompson Research Soft, Thompson Corp., Berkley, CA).

One reviewer $(\mathrm{JG})$ decided whether studies met the inclusion criteria and this was checked by the second reviewer (TX). Using a data collection form, the two reviewers independently extracted data from the included papers on study characteristics and methodological information: that is, the disability identified; which study methods applied; whether or not the sensitivity and cost or cost-effectiveness been analysed. Any differences produced in this process were resolved in a three-way meeting $(\mathrm{JG}, \mathrm{TX}, \mathrm{SH})$. We then further explored the properness of the applications of PRA and KI approaches in the resource-poor countries by analysing their sensitivity, cost-effectiveness, simplicity and rapidness.

\section{Results}

\section{Studies identified}

Eleven studies were identified from the above searches [8-10,16,20,23-28], which used either PRA or KI in the identification process of disability and impairment. We described the features of the 11 papers on study characteristics and methodological information in Table I. Four papers utilised the PRA approach, six papers used the KI method and one paper used both. Five of the 11 papers are comparative studies between the survey approach and either PRA or KI.

\section{Sensitivity}

Four papers addressed the issue of sensitivity on the PRA and KI approaches in the identification of disability $[8,9,20,28]$.

A comparison study involving 430 key informants (village leaders, health workers) plus 670 school children and house-to-house survey of 15,000 households carried out by Pal et al. [9] in West Bengal, India, to detect epilepsy, concluded that the survey method was four times as sensitive as the KI method. In this study, methods were compared for sensitivity and positive predictive value. The survey had an absolute sensitivity of 59\% though their predictive values were similar (36\% vs. 40\%). Another paper [7] also concluded that KI and PRA methods have low sensitivity and cannot provide adequate diagnostic category information or risk factors leading to disability.

Looking at the efficacy of KI and community survey methods for the identification of children with disability in Jamaica, Thorburn et al. [28] utilised 130 key informants and 
house-to-house survey using the 10-question questionnaire. The outcome of this study indicated that the KI method was not sensitive in identifying the disabilities. The house-tohouse survey using 8 community workers screened 5475 children aged 2-9 years with the Ten Questions Questionnaire (TQQ). Of the 821 children that tested positive to the TQQ, 193 were confirmed as having disabilities.

In trying to establish the prevalence of epilepsy in Nakuru, Kenya, Kaamugisha and Feksi [20] used KI and random cluster sample survey methods independently. The KI method proved to be less sensitive than the survey method.

However, other studies have indicated that the PRA or KI approaches had similar prevalence rates compared to house-to-house surveys. In Haryana State in India, Kumar et al. [24] conducted a comparative study utilising KI and house-to-house survey. The results of the prevalence rates were similar with 7.9/1000 and 8.8/1000 for KI and house-to-house survey, respectively. In Kilifi Kenya, the PRA approach had a prevalence rate of 6.9/1000 [10]. This prevalence rate was similar to a previous survey conducted in the same demographic area that had a prevalence rate of 6.1/1000 [5]; however, different children would have been identified as the two studies were conducted at different times (2002 vs. 2004).

In Bangladesh, survey and KI approaches were used to identify children with severe visual impairments or blindness. A total of 1935 children were identified. Of these, $64.3 \%$ were recruited by key informants while the remaining were identified through the survey methodology [25]. The findings indicate that large numbers of children can be recruited through the KI methodology and could have been lost through the survey approaches.

\section{Cost-effectiveness}

Six studies examined the cost-effectiveness of PRA and KI methods. In a study using the PRA approach as a means of identifying children with disabilities in Kilifi Kenya, social mapping as a tool for identification was utilised [10]. A total of 237 children were identified from a population of 3427 children aged between 9 and 15 years drawn from two sublocations in Kilifi. It was estimated in this study that each child identified used approximately US\$1.20. A previous house-to-house survey conducted in the same area was estimated to have used approximately US\$ 7 per child identified [5].

In Bangladesh, Muhit et al. [25] using key informant and survey methods to determine childhood blindness and found out that $25 \%$ of the total cost was specifically needed for the key informant component.

A further comparison study conducted in India [9] employed house-to-house survey and KI methods for ascertaining childhood epilepsy found that the cost of finding one case was US\$ 11 for KI and US\$ 14 for the survey. The cost of finding one successful treatment outcome was US\$ 35 and US\$ 67 for the KI and survey, respectively [9]. The house-to-house survey was conducted on 15,000 households while 430 key informants that included village leaders and health workers together with 670 schoolchildren were utilised in the KI approach.

The survey method was found to be six times more costly than the KI method in a study done in Cameroon [23]. This is a comparative study that was conducted in an urban and rural setting utilising school children as key informants and house-to-house survey to estimate the prevalence and annual incidence of paralytic poliomyelitis.

Kumar et al. [24] using house-to-house survey and village informants found out that the costs for the village informants were about half in comparison with the costs of the house-to- 
house survey. The study population was composed of children aged 0-14 years carried out in 60 villages in India.

In Pakistan, Saeed et al. [26] in a pilot study investigating disabilities among the children using school children as key informants concluded that conventional surveys were more expensive than the KI approach and also that surveys can raise expectations of the parents who are not sure that how to cope with the disability of their children.

\section{Simplicity and rapidness}

Three studies brought out the issue of simplicity and rapidity in the application of PRA and $\mathrm{KI}$ approaches in the process of identification of disability $[16,20,24]$. In a study carried out in Kenya to establish the prevalence of epilepsy in a semi-urban population, Kaamugishaa and Feksi [20] found that the KI method was simpler and more rapid to use than the cluster sample survey approach. They observed that the KI approach could easily be used even by the non-medical workers to identify persons with epilepsy. In a comparative study using house-to-house survey and rapid rural appraisal in South India, Kuruvilla and Joseph [16] highlighted that the analytical simplicity of the PRA. Furthermore, in the study comparing a house-to-house survey with village informants in India, Kumar et al. [24] revealed that the time taken to complete the village informant survey was almost one-third of the house-tohouse survey.

\section{Reveal untreated and other challenging conditions}

Two studies reported that PRA and KI could reveal untreated and other disabling conditions in rural communities $[10,27]$. In a study looking at the prevalence of disabling conditions in rural Northern Thailand using village health communities [27], the author found that there were many people in the community with untreated disabling conditions that could be treated and rehabilitated. These disabling conditions included poliomyelitis, congenital malformations, traumatic amputations and cerebral palsy. In the Kilifi study [10], village leaders, teachers and women's groups were utilised in the identification of children with disabilities, in which the orphans were perceived as being disabled.

\section{Prediction of positive treatment outcome}

We identified one study that showed PRA and KI approaches could predict the success of the treatment outcome [9]. This study was conducted in West Bengal, India and utilised village leaders, health workers and school children as key informants and also conducted a house-to-house survey for 150 households. Predictors of treatment outcomes were calculated by multiple logistic regression analysis, giving adjusted odds ratios for remission. The identification of the disabilities by the key informants was a key process for a precise prediction of the positive treatment outcomes.

\section{Local perceptions of disability}

Local perceptions of disability were a key factor that influenced the identification process of people with disabilities $[10,16]$. According to Gona et al. [10], identification of disability using PRA was influenced by how the local people perceived disability - as a result orphans were perceived as children with disabilities. Similarly, a study [16] in South India highlighted that local perceptions and definition of disability influenced the outcomes of identification of disability. For example people with asthma, cardiac problems, cancer, chronic diarrhea and filariasis were considered disabled, whereas mild disability due to ear and eye infections was rarely considered. 


\section{Discussion}

The results of this review indicate that PRA and KI approaches in the identification of disabilities have both strengths and weaknesses. The unreliable results in the prevalence rates as described $[8,9,20,28]$ could be an indication of weaknesses in the method and illustrate the different perceptions that people have about disability. Such perceptions of disability lead to a different understanding of the concept, which in turn leads to different identification rates of disability in different groups of people with different cultural background.

Cultural norms in a variety of ethnic group settings play a major role in the understanding of disability. For instance, in Kenya, not being able to look after cattle is considered a disability by some tribes (i.e. exclusion from work roles), whereas not being able to be married (i.e. exclusion from social roles) is a serious disability among the Somali people [29] and also among the Yoruba community of Nigeria [29]. This shows the diversity of the concept of disability contributing to the variations of prevalence rates when PRA or KI approaches are utilised in the identification process. The survey approach tends to put more emphasis on the body impairments in which perceived cultural disabilities are not identified. This may explain the increased sensitivity levels but does not address cultural validity. People perceived by certain cultures as disabled will not be picked by the survey method.

The environmental/community perceptions reflected in the information provided through PRA and KIs add richness to the data that increases the validity of the outcome. The information they provide reflects the broad range of disability dimensions as described in the International Classification on Functioning and Disability [1]. This is in contrast to most surveys which tend to focus (to the exclusion of other elements) on only one dimension of disablement, the impairment. The latter reflects a narrow, medical-based understanding of disability that is not compatible both with local cultural perception in low-income countries and also present thinking from the field of disability studies [30,31].

PRA and KI approaches have been highlighted as cheap to use in resource-poor countries $[9,10,23,24]$, using fewer resources than the conventional survey methodology. The use of fewer resources in the application of the PRA and KI methodologies suggest that these approaches could be used in resource-poor countries as an alternative to the conventional surveys, particularly if the process is for service development. Disability surveys themselves should morally and ethically be followed by services for persons with disabilities (PWDs) [32]. In fact, service delivery development for PWDs has often been compromised by the use of resources on surveys that have not been followed up by the development of services. A large portion of these resources could be saved through using PRA and KI as identification methods but this would be at the expense of the decreased sensitivity of impairment.

PRA and KI are seen as easy approaches to implement. They are easy in the sense that they neither need highly trained professionals to carry them out nor a high level of education or literacy to participate. The results reveal that different groups and individuals from the community regardless of their education level or professionalism could take part in the identification process using PRA [16,20,24]. Community involvement in PRA and KI is a key theme in the sustainability disability community development programmes [10].

Results from this review further suggest that PRA and KI approaches can identify untreated and other disabling conditions which the survey methodologies could not identify. As described earlier, a study [27] using KI method identified poliomyelitis, congenital malformation, traumatic amputations and cerebral palsy as treatable conditions that were left untreated. Although their true magnitude might be under-estimated, a significant number of 
these people with these conditions can benefit from medical and other rehabilitation services.

A distinct advantage of PRA and KI approaches in comparison with the survey method is the opportunity of more interaction with the community, developing long-term partnerships and with this the likelihood of increased sustainability. This is not usually observed in survey methods. All these social dynamics have been sighted by a number of studies that applied the PRA or KI methodologies in those countries [10,16,25]. It is observed that these key informants could be empowered to reduce social stigma, increase awareness and improve health-seeking behaviour of community members.

PRA and KI approaches may not provide accurate prevalence rates, but identify PWDs whom the community thinks require support. PRA and KI may underestimate the prevalence by missing cases, however if used systematically to cover a large population over a period of time, these approaches can provide an opportunity for developing national and local database of PWDs at little cost. Such databases will be of enormous value for advocacy, research and programme development for PWDs [25].

PRA could be useful approach by which PWDs themselves can evaluate and prioritise their needs and plan for future social action. It can be a powerful tool for advocating and lobbying for disability rights in resource-poor countries.

However, there are limitations associated with PRA and KI approaches. They tend to under enumerate disabilities, especially those that are less physical. Relying on the knowledge of the local people is another aspect in these approaches that could affect the validity of the results. The perceptions of disability by different communities influence the identification process and lead to inaccurate identification.

The strengths of PRA and KI lie in the simplicity of the processes involved using interviews and mapping. They have been documented to be cheap and fast in the identification of disability. Their participatory nature involving local people within the community tends to raise awareness and empower the people to seek local solutions to the challenges faced by disabled people and their families.

\section{Conclusions}

The use of PRA and KI approaches in the identification of people with disability in resource-poor countries are useful and practical options. They do however have less sensitivity for detecting impairments in comparison with the survey method. They identify PWDs according to local perceptions of disability, whereas surveys focus on the impairment dimension of disability and frame the understanding of disability around conceptions that originate in other cultures and continents and are not compatible with present thinking in the field of disability studies. PRA and KI are less costly than surveys; they are simple and more rapid. It appears better to use the survey method if the identification of PWDs is focusing on impairment and is seeking statistical results. If the identification is focusing on disability and is for service development to support PWDs and their families, then PRA or KI approaches could be a more appropriate method, cheaper and a more community empowering and sustainable option.

\section{Acknowledgments}

We would like to thank the director of Kenya Medical Research Institute (KEMRI) for permission to publish these data. This work is supported by KEMRI, Wellcome Trust Collaborative research programme and C. P. Charitable Trust Fund (UK). Dr Charles Newton is supported by the Wellcome Trust, UK. 


\section{References}

1. WHO. International classification of functioning, disability and health. World Health Organisation; Geneva, Switzerland: 2001.

2. Al-Ansari A. Prevalence estimates of physical disability in Bahrain - a household survey. Int Disabil Stud. 1989; 11:21-24. [PubMed: 2527840]

3. Bezzaoucha A, Dekkar N. Handicaps in Algiers according to a household survey. Int J Epidemiol. 1990; 19:466-471. [PubMed: 2142931]

4. Hardas U, Waikar A. Pilot survey of disabled children in and around Nagpur. Indian J Pediatr. 1974; 41:267-271. [PubMed: 4280382]

5. Mung'ala-Odera V, Meehan R, Njuguna P, Mturi N, Alcock K, Carter JA, Newton CR. Validity and reliability of the 'Ten Questions' questionnaire for detecting moderate to severe neurological impairment in children aged 6-9 years in rural Kenya. Neuroepidemiology. 2004; 23:67-72. [PubMed: 14739570]

6. Bharucha NE, Bharucha EP, Dastur HD, Schoenberg BS. Pilot survey of the prevalence of neurologic disorders in the Parsi community of Bombay. Am J Prev Med. 1987; 3:293-299. [PubMed: 3330662]

7. Das S, Sanyal K, Moitra A. A pilot study on neuroepidemiology in urban Bengal. Indian J Public Health. 1998; 42:34-36. [PubMed: 10389506]

8. Mung'ala-Odera V, Newton CR. Identifying children with neurological impairment and disability in resource-poor countries. Child Care Health Dev. 2007; 33:249-256. [PubMed: 17439437]

9. Pal DK, Das T, Sengupta S. Comparison of key informant and survey methods for ascertainment of childhood epilepsy in West Bengal, India. Int J Epidemiol. 1998; 27:672-676. [PubMed: 9758124]

10. Gona JK, Hartley S, Newton CR. Using participatory rural appraisal (PRA) in the identification of children with disabilities in rural Kilifi, Kenya. Rural Remote Health. 2006; 6:553. [PubMed: 16956314]

11. Bar-On A, Prinsen G. Planning, communities and empowerment: an introduction to participatory rural appraisal. Int Social Work. 1999; 42:277-294.

12. Pretty J. Alternative systems of inquiry for sustainable agriculture. IDS Bull. 1994; 25:37-38.

13. Groce NE. Disability in cross-cultural perspective: rethinking disability. Lancet. 1999; 354:756757. [PubMed: 10475203]

14. Rifkin SB. Rapid rural appraisal: its use and value for health planners and managers. Public Admin. 1996; 74:509-526.

15. Kuipers P, Kendall E, Hancock T. Developing a rural community-based disability service: (I) service framework and implementation strategy. Aust J Rural Health. 2001; 9:22-28. [PubMed: 11703263]

16. Kuruvilla S, Joseph A. Identifying disability: comparing house-to-house survey and rapid rural appraisal. Health Policy Plan. 1999; 14:182-190. [PubMed: 10538721]

17. Eyler AA, Mayer J, Rafii R, Housemann R, Brownson RC, King AC. Key informant surveys as a tool to implement and evaluate physical activity interventions in the community. Health Educ Res. 1999; 14:289-298. [PubMed: 10387507]

18. Warheit GJ, Buhl JM, Bell RA. A critique of social indicators analysis and key informants surveys as needs assessment methods. Eval Program Plan. 1978; 1:239-247.

19. Morrissey JP, Ridgely MS, Goldman HH, Bartko WT. Assessments of community mental health support systems: a key informant approach. Community Ment Health J. 1994; 30:565-579. [PubMed: 7835042]

20. Kaamugisha J, Feksi AT. Determining the prevalence of epilepsy in the semi-urban population of Nakuru, Kenya, comparing two independent methods not apparently used before in epilepsy studies. Neuroepidemiology. 1988; 7:115-121. [PubMed: 3136404]

21. McDonald JR, Natarajan T. Community care needs of people with AIDS - the key informant study: a research method for policy development, service planning, and achieving consensus. $\mathbf{J}$ Palliat Care. 1989; 5:16-19. [PubMed: 2746430]

22. NHSCRD. CRD Report Number 4: NHS Centre for Reviews and Dissemination. 2nd ed. University of York; York: 2001. 
23. Heymann DL. House-to-house and school lameness surveys in Cameroon: a comparison of two methods for estimating the prevalence and annual incidence of paralytic poliomyelitis. Rev Infect Dis. 1984; 6(Suppl 2):S376-S378. [PubMed: 6740076]

24. Kumar R, Singh A, Kumar V. Survey of village informants - an alternate method to estimate paralytic poliomyelitis prevalence in rural area. Indian J Pediatr. 1991; 58:239-243. [PubMed: 1879905]

25. Muhit MA, Shah SP, Gilbert CE, Hartley SD, Foster A. The key informant method: a novel means of ascertaining blind children in Bangladesh. Br J Ophthalmol. 2007; 91:995-999. [PubMed: 17431019]

26. Saeed K, Wirz S, Gater R, Mubbashar MH, Tomkins A, Sullivan K. Detection of disabilities by schoolchildren: a pilot study in rural Pakistan. Trop Doct. 1999; 29:151-155. [PubMed: 10448238]

27. Swaddiwudhipong W, Amaritchavarn V, Boonyabuncha S. Prevalence of disabling conditions in a rural northern Thai community: a survey conducted by village health communicators. Southeast Asian J Trop Med Public Health. 1994; 25:45-49. [PubMed: 7825025]

28. Thorburn MJ, Desai P, Durkin M. A comparison of efficacy of the key informant and community survey methods in the identification of childhood disability in Jamaica. Ann Epidemiol. 1991; 1:255-261. [PubMed: 1669506]

29. Helander, B. Disability as incurable illness: health, process and personhood in Southern Somalia. In: Ingstad, B.; Whyte, S., editors. Disability and culture. University of California Press; London: 1995.

30. Hammell, KW. Perspectives on disability and rehabilitation: contesting assumptions, challenging practice. Churchill Livingstone; Edinburgh: 2006.

31. Oliver, M. Understanding disability: from theory to practice. St. Martin's Press; New York: 1996.

32. Miles S. Engaging with the Disability Rights Movement: the experience of community-based rehabilitation in southern Africa. Disabil Soc. 1996; 11:501-518. 


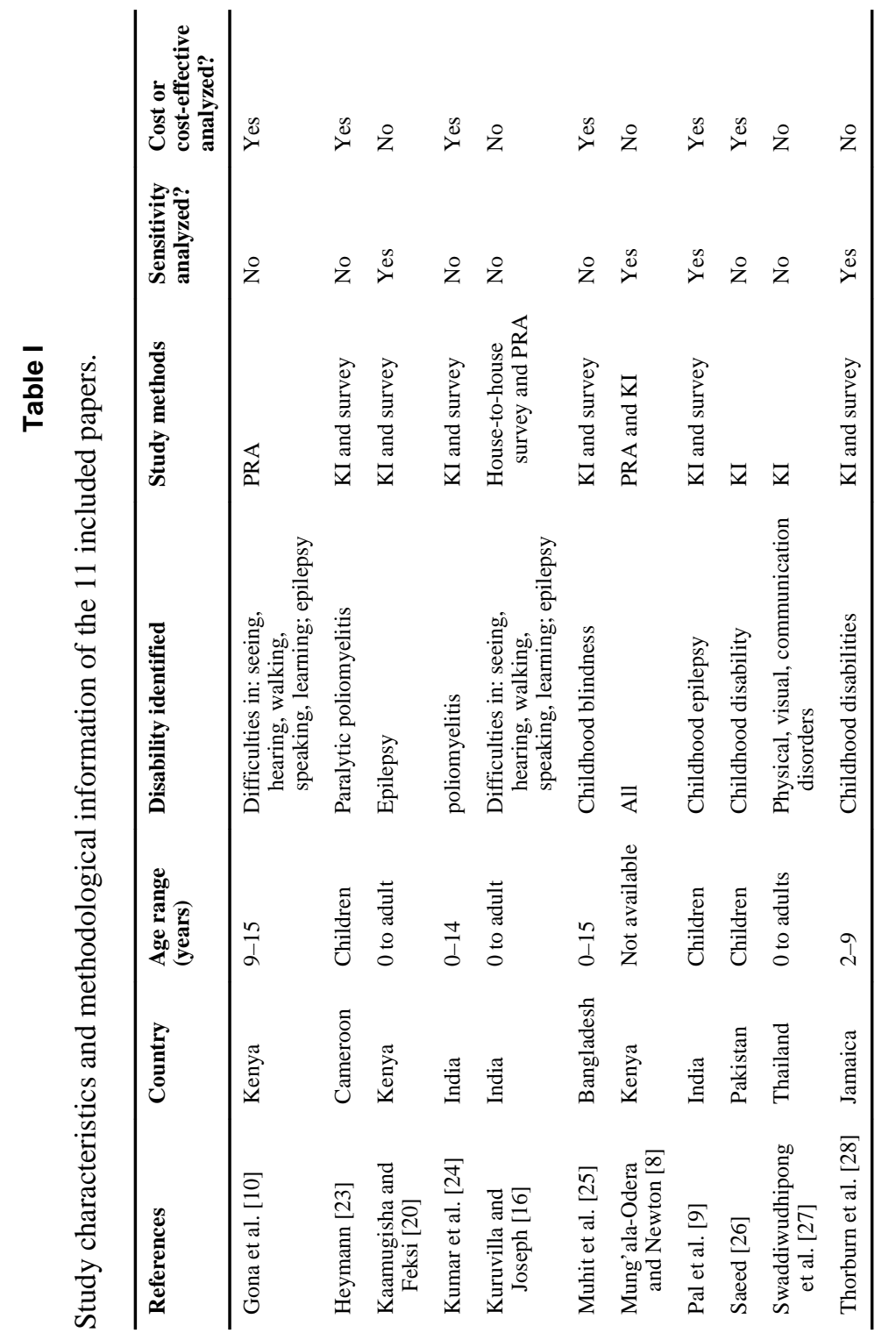

